\title{
AN APPLICATION OF NEVANLINNA-PÓLYA THEOREM TO A COSINE FUNCTIONAL EQUATION
}

\author{
HIROSHI HARUKI
}

(Received 3 February 1969)

Communicated by J. B. Miller

\section{Introduction}

We consider the cosine functional equation (see $[1,2,3])$

$$
f(0)(f(x+y)+f(x-y))=2 f(x) f(y),
$$

where $f(z)$ is an entire function of a complex variable $z$ and $x, y$ are complex variables.

It is clear that the only entire solution of (1) is $a \cos b z$ where $a, b$ are arbitrary complex constants.

In Section 2 we shall prove the following

THEOREM 1. If $f(z)$ is an entire function of a complex variable $z$, then (1) implies the following functional equation (2) with some $g$ :

$$
\begin{aligned}
|f(0)|^{2}\left(|f(x+y)|^{2}+|f(x-y)|^{2}\right)= & 2|f(x)|^{2}|f(y)|^{2} \\
& +2|g(x)|^{2}|g(y)|^{2},
\end{aligned}
$$

where $g(z)$ is an entire function of a complex variable $z$.

In Section 3 we shall prove a converse of Theorem 1, i.e., the following

THEOREM 2. If $f(z), g(z)$ are entire functions of a complex variable $z$, then (2) implies (1).

To this end we shall use the following

THEOREM A. If $f(z), g(z), h(z), k(z)$ are entire functions of a complex variable $z$ and satisfy $|f(z)|^{2}+|g(z)|^{2}=|h(z)|^{2}+|k(z)|^{2}$ in $|z|<+\infty$, then there exists $a$ unitary matrix $\left(\begin{array}{ll}a & \beta \\ \gamma & \delta\end{array}\right)$ such that

$$
\begin{aligned}
& h(z)=\alpha f(z)+\beta g(z) \\
& k(z)=\gamma f(z)+\delta g(z)
\end{aligned}
$$

in $|z|<+\infty$, where $\alpha, \beta, \gamma, \delta$ are complex constants.

Proof. See $[4,5]$. 
COROLLARY OF THEOREM A. If $f(z), g(z), h(z), k(z)$ are entire functions of $z$ and satisfy $\left|f^{\prime}(z)\right|^{2}+\left|g^{\prime}(z)\right|^{2}=\left|h^{\prime}(z)\right|^{2}+\left|k^{\prime}(z)\right|^{2}$ in $|z|<+\infty$ and if $f(0)=g(0)=$ $h(0)=k(0)=0$, then $|f(z)|^{2}+|g(z)|^{2}=|h(z)|^{2}+|k(z)|^{2}$ in $|z|<+\infty$.

Proof. By the hypothesis and by Theorem $\mathrm{A}$ there exists a unitary matrix $\left(\begin{array}{ll}a & \beta \\ \gamma & \delta\end{array}\right)$ such that

$$
\begin{aligned}
& h^{\prime}(z)=\alpha f^{\prime}(z)+\beta g^{\prime}(z) \\
& k^{\prime}(z)=\gamma f^{\prime}(z)+\delta g^{\prime}(z)
\end{aligned}
$$

in $|z|<+\infty$, where $\alpha, \beta, \gamma, \delta$ are complex constants.

By (3), (4) and by $f(0)=g(0)=h(0)=k(0)=0$ we have

$$
\begin{aligned}
& h(z)=\alpha f(z)+\beta g(z) \\
& k(z)=\gamma f(z)+\delta g(z) .
\end{aligned}
$$

Since $(\alpha \beta / \gamma \delta)$ is a unitary matrix, by (5), (6) the Corollary is proved.

By Theorems 1,2 we have the following

THEOREM 3. The only system of entire solutions of (2) is $f(z)=a \cos b z$, $g(z)=a \exp (i \theta) \sin b z$ where $a, b$ are arbitrary complex constants and $\theta$ is an arbitrary real constant.

Proof. It is clear from Theorems $1,2$.

\section{Proof of Theorem 1}

We may assume that $f(z)$ ₹ const. Otherwise the proof is clear.

Differentiating both sides of (1) twice with respect to $y$ and putting $y=0$, we have

$$
f(0) f^{\prime \prime}(x)=f^{\prime \prime}(0) f(x) .
$$

Differentiating both sides of (1) with respect to $x$ and then with respect to $y$, we have

$$
f(0)\left(f^{\prime \prime}(x+y)-f^{\prime \prime}(x-y)\right)=2 f^{\prime}(x) f^{\prime}(y) .
$$

We can deduce that $f^{\prime \prime}(0) \neq 0$. Otherwise, by (1), (7) $f(z)$ is a complex constant, contradicting the assumption that $f(z)$ ₹ const.

By (7), (8) we have

$$
f(0)(f(x+y)-f(x-y))=2 \frac{f(0)}{f^{\prime \prime}(0)} f^{\prime}(x) f^{\prime}(y) .
$$

(1), (9) and the parallelogram identity $|a+b|^{2}+|a-b|^{2}=2|a|^{2}+2|b|^{2}(a, b$ complex) yield that 
(10)

$$
\begin{aligned}
|f(0)|^{2}\left(|f(x+y)|^{2}\right. & \left.+|f(x-y)|^{2}\right) \\
& =2|f(x)|^{2}|f(y)|^{2}+2\left|\frac{f(0)}{f^{\prime \prime}(0)}\right|^{2}\left|f^{\prime}(x)\right|^{2}\left|f^{\prime}(y)\right|^{2} .
\end{aligned}
$$

By (10) we see that (1) implies (2) with $g(z)=\sqrt{f(0) / f^{\prime \prime}(0)} f^{\prime}(z)$ which is an entire function of a complex variable $z$. Q.E.D.

\section{Proof of Theorem 2}

Upon putting $x=y=0$ in (2), we see that

$$
g(0)=0 .
$$

We next take Laplacians $\partial^{2} / \partial s^{2}+\partial^{2} / \partial t^{2}$ of both sides of (2) with respect to $y=s+i t(s, t$ real $)$ and obtain

$$
\begin{aligned}
& |f(0)|^{2}\left(4\left|f^{\prime}(x+y)\right|^{2}+4\left|f^{\prime}(x-y)\right|^{2}\right) \\
& =8|f(x)|^{2}\left|f^{\prime}(y)\right|^{2}+8|g(x)|^{2}\left|g^{\prime}(y)\right|^{2},
\end{aligned}
$$

or

$$
\begin{aligned}
& |f(0)|^{2}\left(\left|f^{\prime}(x+y)\right|^{2}+\left|f^{\prime}(x-y)\right|^{2}\right) \\
& =2|f(x)|^{2}\left|f^{\prime}(y)\right|^{2}+2|g(x)|^{2}\left|g^{\prime}(y)\right|^{2},
\end{aligned}
$$

since, by [6], $\Delta|f|^{2}=4\left|f^{\prime}\right|^{2}$.

When $x$ is arbitrarily fixed, $f(0)(f(x+y)-f(x)), f(0)(f(x-y)-f(x))$, $\sqrt{2} f(x)(f(y)-f(0)), \sqrt{2} g(x) g(y)$ are entire functions with

$$
\begin{aligned}
(f(0)(f(x+y)-f(x)))_{y=0} & =(f(0)(f(x-y)-f(x)))_{y=0} \\
& =(\sqrt{2} f(x)(f(y)-f(0)))_{y=0}=(\sqrt{2} g(x) g(y))_{y=0}=0
\end{aligned}
$$

(by (11)). Moreover, by (12) we have in $|y|<+\infty$

$$
\begin{aligned}
\mid \frac{\partial}{\partial y}(f(0)(f(x+y)- & f(x)))\left.\right|^{2}+\left|\frac{\partial}{\partial y}(f(0)(f(x-y)-f(x)))\right|^{2} \\
= & \left|\frac{\partial}{\partial y}(\sqrt{2} f(x)(f(y)-f(0)))\right|^{2}+\left|\frac{\partial}{\partial y}(\sqrt{2} g(x) g(y))\right|^{2}
\end{aligned}
$$

Hence, by Corollary of Theorem A we have in $|y|<+\infty$

$$
\begin{aligned}
& \begin{aligned}
&\left.f(0)\right|^{2}\left(|f(x+y)-f(x)|^{2}+|f(x-y)-f(x)|^{2}\right) \\
&= 2|f(x)|^{2}|f(y)-f(0)|^{2}+2|g(x)|^{2}|g(y)|^{2} .
\end{aligned}
\end{aligned}
$$

Subtracting (13) from (2) and using the identity $|a-b|^{2}=|a|^{2}+|b|^{2}-$ $2 \operatorname{Re}(a b)$, we see that

$$
|f(0)|^{2} \operatorname{Re}((f(x+y)+f(x-y)) \overline{f(x)})=2|f(x)|^{2} \operatorname{Re}(f(y) \overline{f(0)}) .
$$


We may assume that $f(0) \neq 0$. Otherwise the proof is clear. Hence, by the continuity of $f$ there exists a neighborhood $V$ of the origin where $f(x) \neq 0$.

So, by (14) we have in $V$ and for every complex $y$

$$
\operatorname{Re}\left(\frac{1}{f(x)}(f(x+y)+f(x-y))-2 \frac{1}{f(0)} f(y)\right)=0 .
$$

Since $f(z)$ is an entire function of a complex variable $z$, by (15) we have in $V$ and for every complex $y$

$$
\frac{1}{f(x)}(f(x+y)+f(x-y))-2 \frac{1}{f(0)} f(y)=C,
$$

where $C$ is a complex constant.

Upon putting $y=0$ in (16), we see that

$$
C=0 .
$$

By (16), (17) and by the Identity Theorem we have (1).

Q.E.D.

\section{References}

[1] A. L. Cauchy, Cours d'Analyse (Paris, 1821; Oeuvres Complètes (2), 3 (1897), 106-113).

[2] T. M. Flett, 'Continuous solutions of the functional equation $f(x+y)+f(x-y)=2 f(x) f(y)$ ', Amer. Math. Monthly 70 (1963), 392.

[3] S. Kaczmarz, 'Sur l'équation fonctionnelle $f(x)+f(x+y)=\varphi(y) f(x+y / 2)$ ', Fund. Math. 6 (1924), $122-129$.

[4] R. Nevanlinna-G. Pólya, 'Unitäre Transformationen analytischer Funktionen', Jahresbericht der deutschen Mathematiker-Vereinigung 40 (1931) 80. (Aufgabe 103).

[5] H. Schmidt, 'Lösung der Aufgabe 103', Jahresbericht der deutschen Mathematiker-Vereinigung 43 (1934), 6-7.

[6] Pólya, G., u. G. Szegö, Aufgaben und Lehrsätze aus der Analysis (I, S. 94. Berlin-GöttingenHeidelberg, Springer Verlag 1954).

Faculty of Mathematics

University of Waterloo

Waterloo, Ontario, Canada 